Journal of Computer Science 7 (10): 1458-1464, 2011

ISSN 1549-3636

C 2011 Science Publications

\title{
Evaluation of Pressure-Slippage-Generating Tactile Mouse using Edge Presentation
}

\author{
Yiru Zhou, Xuecheng Yin and Masahiro Ohka \\ Department of Complex Systems Science, Graduate School of Information Science, \\ Nagoya University, Furo-cho, Chikusa-ku, Nagoya 464-8601, Japan
}

\begin{abstract}
Problem statement: Although a conventional tactile display can generate distributed pressure, our tactile mouse can generate not only distributed pressure but also slippage force. In previous works, this tactile mouse was effective on virtual figure presentation tests. Advanced tests are required to evaluate this tactile display. Approach: To compare virtual reality presented by the present tactile mouse and a real object, we produced an edge presentation apparatus composed of an x-y stage and two rotary stages to compare real feeling with virtual reality. In virtual edge presentation, slippage stimulus of a spike was generated in addition to step-height stimulus when a mouse cursor travels on the virtual edge. Results: In virtual edge presentation, we performed three series of experiments: distributed pressure presentation, combined presentation of distributed pressure and vertical slippage force and combined presentation of distributed pressure and parallel slippage force. In the case of the combined presentation of distributed pressure and parallel slippage force, a human subject's judgment of edge direction was performed with the highest precision among virtual edge presentation tests. When the human subject touched the real edge orientation presented by the apparatus, the precision became higher than any case of virtual presentation. Conclusion/Recommendations: The combined presentation of distributed pressure and parallel slippage force was effective to present a virtual edge. The pressure-slippage-generating tactile mouse can enhance the degree of reality of a virtual figure.
\end{abstract}

Key words: Virtual reality, psychophysics, tactile mouse, pressure, slippage force, edge presentation, fast adapting, slowly adapting, tactile organs, linear motor, USB interface, figure presentation, slippage-generating

\section{INTRODUCTION}

Virtual Reality (VR) technology and tele-existence (Tachi, 1992) have recently progressed in the research fields of telecommunication and tele-robotics. In these fields, several mechanisms have been tentatively presented for tactile displays. Since VR requires virtual object handing, researchers have paid particular attention to tactile displays (Salisbury and Srinivasan, 1997; Shinohara et al., 1998; Shimojo et al., 1999; Konyo et al., 2000; Iwata et al., 2001; Nara et al., 2001; Arata and Ikei, 2006; Ohka et al., 2007; Zhou et al., 2010). On the other hand, the mechanoreceptive units of human tactile organs are known as the Fast Adapting type I unit (FA I), the Fast Adapting type 2 unit (FA II), the Slowly Adapting type I unit (SA I) and the Slowly Adapting type II unit (SA II). Since four kinds of mechanoreceptive units exist in the skin, an ideal tactile display requires a mechanism to stimulate them (Bolanowaski et al., 1988).
In a preceding study (Zhou et al., 2010), we developed a new tactile display mounted on a mouse capable of presenting not only distributed pressure but also tangential force to stimulate the four kinds of mechanoreceptive units. The mouse is equipped with a bimorph-piezoelectric-actuator array and a twodimensional electro-magnetic-linear motor to present pressure and slippage forces, respectively. To evaluate the mouse's presentation accuracy, we performed a series of experiments that traced the virtual figure contours. The distributed pressure display stimulates FA I, FA II and SA I, while the $x-y$ linear motor stimulates SA II. We evaluated the deviation from the desired trajectory for each virtual figure to confirm the effect on the present combined stimulus. To evaluate this tactile mouse, we performed edge tracing tests for virtual circles, triangles and squares with four male subjects. The experimental results showed that presentation capability was enhanced with the combined stimulation because the edge tracing precision obtained using combined stimulation

Corresponding Author: Masahiro Ohka, Department of Complex Systems Science, Graduate School of Information Science, Nagoya University, Furo-cho, Chikusa-ku, Nagoya 464-8601, Japan 
exceeded that using only pressure stimulation. In the case that obtained highest enhancement, the error of tracing is reduced to around $1 / 3$. However, we should investigate what combination of pressure and slippage force is appropriate to generate virtual objects.

In this study we examine the influence of combination of pressure and slippage force on distinguishing edge orientation because any figure is composed of several edges. While the preceding study examined the tracing precision, the present study examines the psychological effect on virtual figure presentation to check whether the human subject can distinguish the figure or not. We adopt psychophysics as the experimental procedure to evaluate the distinction.

To compare virtual reality presented by the present tactile display and a real object, we produced an edge presentation apparatus composed of an $\mathrm{x}-\mathrm{y}$ stage and two rotary stages to compare real feeling with virtual reality. In virtual edge presentation, the tangential stimulus of a spike is generated in addition to step-height stimulus when a mouse cursor travels on the virtual edge.

In a series of psychophysical experiments, human subjects palpated two virtual edges: a standard edge fixed during the entire experiment and a comparison edge that could be changed. Human subjects try to change the orientation of the comparison edge to make it coincide with the orientation of the standard edge. From this method we obtained a threshold, which we assumed to represent the sensitivity of human sensation. By evaluating changes in the threshold, we determined the optimum combination of distributed pressure and tangential force. Furthermore, we evaluated the degree of reality presented by the tactile display with comparison between the thresholds of the virtual edge test and real edge test.

\section{MATERIALS AND METHODS}

Real edge orientation-generating apparatus: In this study we compare virtual reality presented by the present tactile display and a real object. Thus, we produced an edge presentation apparatus composed of an $x-y$ stage and two rotary stages to compare real feeling with virtual reality as shown in Fig. 1(a). This apparatus is composed of an orthogonal robot (NSK, XY-HD3080-703-001; positioning resolution: $5 \mu \mathrm{m}$ ) and two motorized rotary stages (Chuo Precision Industrial Co., ARS-636-HM; angular resolution: 0.008). A step-height stimulus of aluminum alloy is mounted on each rotary stage. The step-height is 400 $\mu \mathrm{m}$ as shown in Fig. 1(b). Human subjects can adjust the orientation of the edge by operating the controller with their left hand. The set up for edge adjustment is shown in Fig. 2.

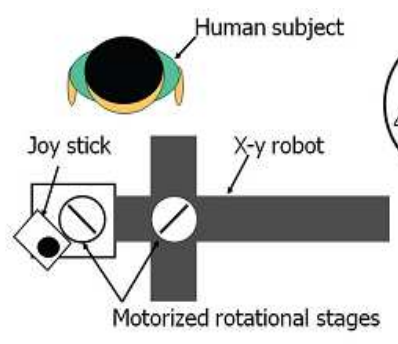

(a) Top view

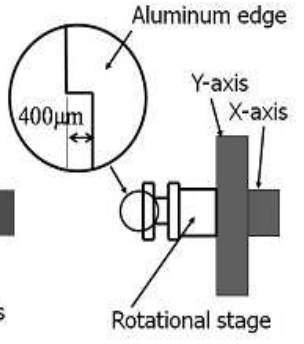

(b) Side view
Fig. 1: Real edge presentation apparatus

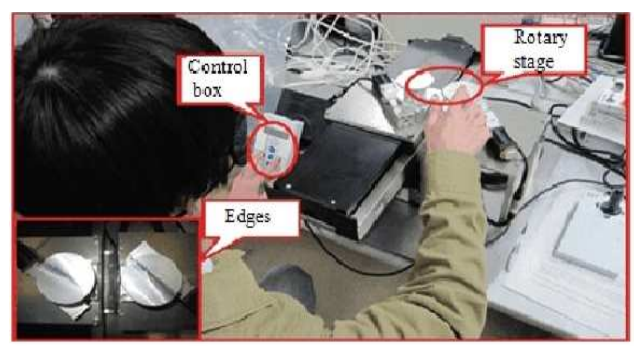

Fig. 2: Photograph of real edge adjustment

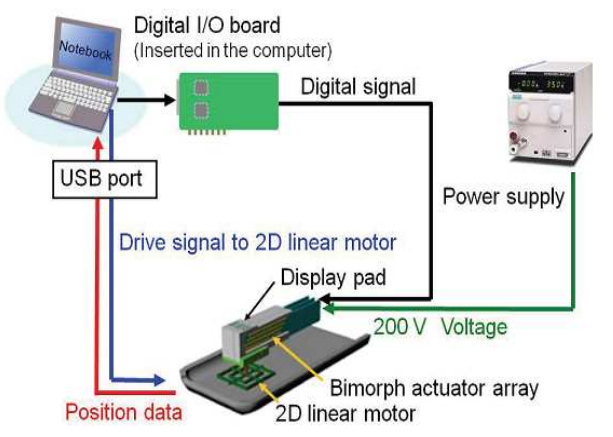

Fig. 3: Schematic diagram of tactile mouse system

Tactile display: In a previous study, we developed a tactile display capable of presenting distributed pressure and slippage. Since we adopt the display as a tactile presentation system, we introduce the tactile display first. A block diagram for controlling the tactile mouse is shown in Fig. 3. The $x$ and $y$-directional position data of the mouse cursor are sent to a computer through a USB interface to calculate the output stimuli of the distributed pressure and the tangential forces on the basis of the positioning relationship between the mouse cursor and the virtual object. The stimulus pins of the display pad are driven by the driver boards for the piezoelectric actuator installed on the Braille dot cell controlled by the Digital Input Output (DIO) board to generate the specified pin protruding pattern. The signals for the tangential force are transmitted to a motor control board installed in the mouse through a USB interface to generate slippage force. 
In this tactile display, the distributed pressure and slippage force are generated by the Braille dot cell (SC2, KGS, Co.; http://www.kgs-jpn.co.jp/epiezo.html) and the linear motor, respectively. Since the piezoelectric ceramic actuator in the SC2 is a bimorph type, its motion is controlled by the voltage applied to the core electrode. When source voltage $200 \mathrm{~V}$ is applied between the upper and bottom electrodes, the end of the actuator bends downward if $200 \mathrm{~V}$ is applied to the core electrode. If $0 \mathrm{~V}$ is applied, the end of the actuator bends upward. The stroke of the upward and downward motion is about $1 \mathrm{~mm}$. The strength of the force generated by each actuator is around $0.06 \mathrm{~N}$. The characteristics of the actuator are shown in Fig. 4. The stimulus pin is moved up-and-down based on the upward and downward bending of the piezoelectric ceramic actuator. Since we used three Braille dot cells, a dot pattern is formed on the display pad the piezoelectric ceramic actuator. Since we used three Braille dot cells, a dot pattern is formed on the display pad of $4 \times 6$ pins. Although the force of $0.06 \mathrm{~N}$ generated by each actuator seems to be small, the Braille dot cell can generate force up to $1.4 \mathrm{~N}$ because it is composed of 24 actuators.

To generate slippage force, we used Fuji Xerox's tactile mouse. This mouse is equipped with an $x-y$ linear motor on an optical mouse to generate static shearing force and its vibration. Force signals are transmitted to a motor control board installed in the mouse through the USB interface. The characteristics of the linear motor are shown in

Although the linear motor generates tangential force of $0.7 \mathrm{~N}$, the force is reduced when the tactile display is mounted on the motor as shown in Fig. 5. This reduction is caused by bending resistance of wiring of the tactile display.

The present tactile mouse is developed by combining the distributed pressure display and Fuji Xerox's tactile mouse. If user fingers are placed on the tactile mouse's display pad and the mouse is moved on the virtual texture, users can feel a convex or concave surface based on the virtual texture. Additionally, the user feels marked slippage force when the mouse cursor travels on a high coefficient of friction.

Advantage of psychophysical experiment for evaluation of tactile display: Although we have to evaluate how the subject feels the virtual tactile sensation generated by the present tactile display, the feeling is an individual event caused in the subject's mind. Since psychophysics introduces experimental procedures that relate events caused in the human mind to an external physical stimulus, we use psychophysics or evaluation of the tactile display. In psychophysics, several procedures have been introduced (Gescheider, 1997). Since an adjustment method is effective to obtain the difference threshold of human sensation, we adopted the adjustment method as a psychophysical experiment.

Adjustment method: In the adjustment method, a pair of stimuli is presented. One stimulus is not changed during the experiment and is called the standard stimulus. The other is adjusted by the subject and is called the comparison stimulus. Human subjects try to adjust the comparison stimulus to obtain same tactile sensation between the two stimuli. Since subjects can participate actively the experiment, this method prevents them from becoming bored.

In both the real edge test and virtual edge test, human subjects palpated the standard edge and the comparison edge. In the real edge test, they adjusted the orientation of the comparison edge to make it the same orientation as the standard edge using the control box of the real edge presentation apparatus. In the virtual edge test, they also adjusted the orientation of the comparison edge in virtual reality to make it the same orientation as the standard edge in virtual reality.

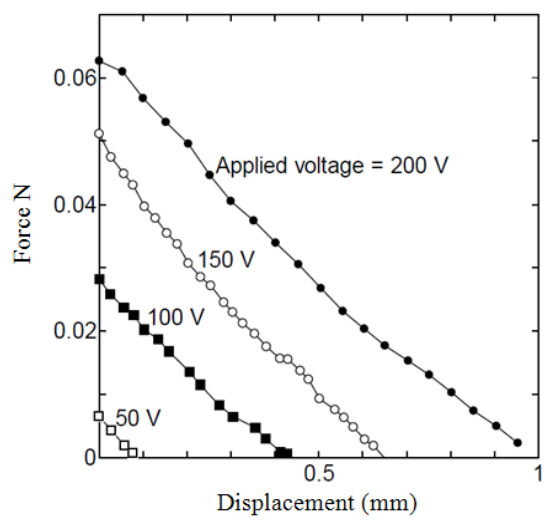

Fig. 4: Characteristics of bimorph piezoelectric actuator

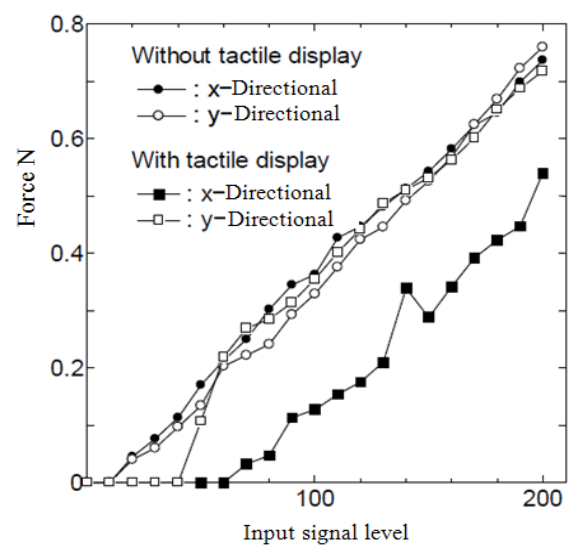

Fig. 5:Characteristics of shearing force generation of tactile mouse 
Concept of virtual edge presentation: When we palpate a relief-like object, we try to explore the edges of the object. At that time, we slide a finger from the background flat surface to the edge to feel tangential force as shown in Fig. 6. While we feel compressive force generated by the convex portion of the object, we feel tangential force generated by scratching against the edge. The tangential force generated on the side of the virtual object is decomposed into vertical and parallel components. In this study, we treat these three components of force.

Edge presentation using tactile mouse: The tactile display and the linear motor are controlled to generate the three components of force. The display pad is superimposed in the virtual figure in Fig. 7. To generate distributed pressure, pins are protruded according to the direction of the edge of the virtual figure. To generate the scratching feeling caused by passing the edge, tangential force is generated when the display pad just passes the edge. The variation in tangential force is one pulse whose amplitude is around $0.4 \mathrm{~N}$. Although resultant force of vertical and parallel components is generated in the actual touching, extreme cases of only the vertical component and only the parallel component are examined in this study.

In the virtual edge test, the subjects also adjust the orientation of the comparison edge in virtual reality to make it the same orientation as the standard edge. This adjustment of edge orientation of the comparison stimulus is performed by specific keys of the computer keyboard. Furthermore, edges on the main panel are hidden by filled circles as shown in to prevent from showing the edge orientation to the human subjects. If the cursor is under the circle, the color of the circle changes from black to blue to show which circle includes the cursor.

Incorporation of adjustment method: The adjustment method for virtual reality is achieved by special software we produced. Figure 8 shows the main panel of this software. The left and right edges are standard and comparison stimuli, respectively. The reason for the circle is to produce a condition similar to that of the real edge. The human subject manipulates the tactile mouse as shown in Fig. 9 to recognize the orientation of both edges. The human subject can transport the mouse between two circles to compare two edges. This cursor transportation is accomplished by pushing specific keys.

Experimental condition of Real edge test : This test was performed using the real edge presentation apparatus. We adoptedseven stimuli, 0, 15, 30, 45, 60, 75 and $90^{\circ}$. As the standard stimulus in this test. We performed 10 trials for every standard stimulus, which was presented on the left in half of them and on the right in the others.

As a result, 70 trials were randomly performed for each human subject. In this experiment, five male persons participated. Their age was around 26. Every human subject wore an eye mask.

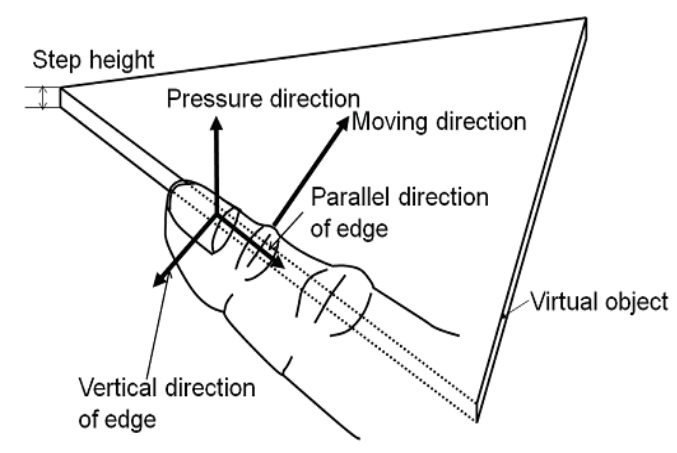

Fig. 6: Way of palpating relief-like object

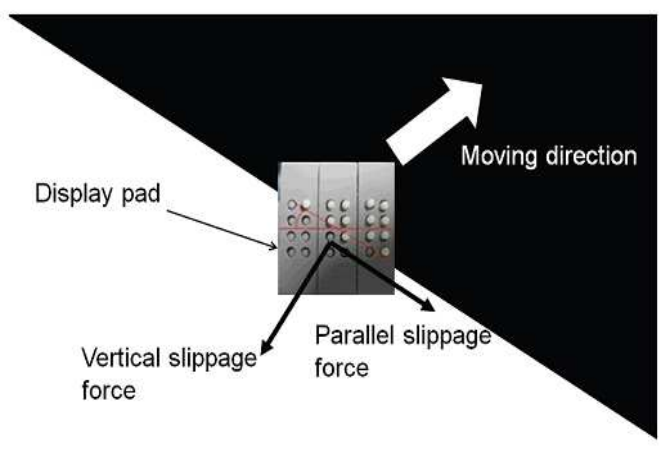

Fig. 7: Edge presentation using tactile mouse

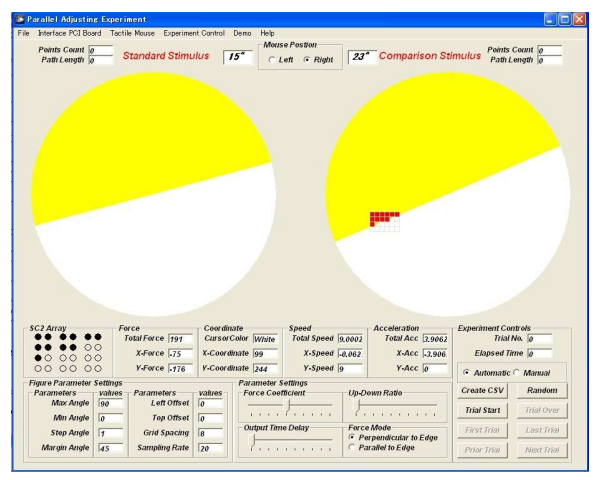

Fig. 8: Edge presentation using tactile mouse (hidden panel) 


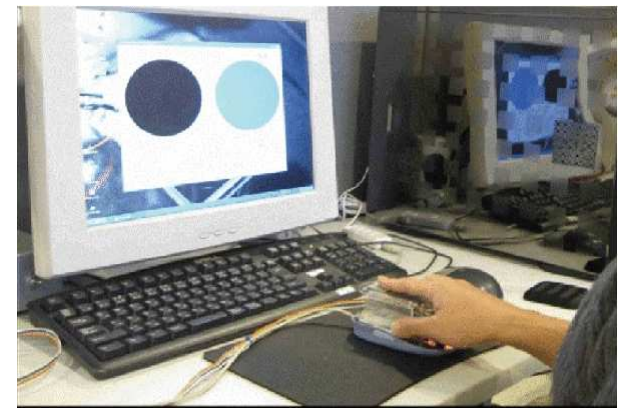

Fig. 9: Scene of virtual edge test

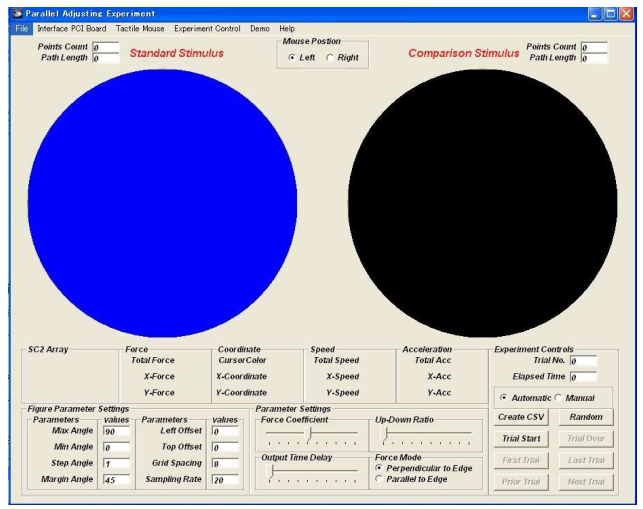

Fig. 10: Main panel during experiment

Experimental condition of virtual edge test: This test was performed using the tactile mouse and the presentation software shown in Fig. 8. In this test, five male persons participated. To compare this result with the real edge test's result, we adopted the same conditions as the real edge test regarding standard stimuli and trial number. However, human subjects did not wear the eye mask in this test because edges were hidden by the filled circles in Fig. 10. To examine which combination of pressure and slippage force is effective for edge presentation, we performed the following tests.

- Only distributed pressure is presented

- Distributed pressure and slippage force directed to the vertical direction of the edge are presented

- Distributed pressure and slippage force directed to the parallel direction of the edge are presented

\section{RESULTS}

Real edge test: Figure 11 shows the experimental result obtained from the real edge apparatus. The dotted line shows the ideal case, in which the comparison edge direction is adjusted completely to the standard edge direction.

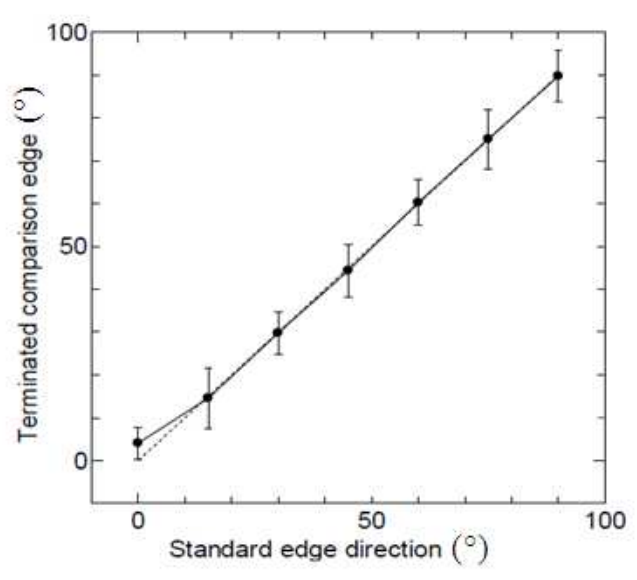

Fig. 11: Experimental result obtained from real edge test

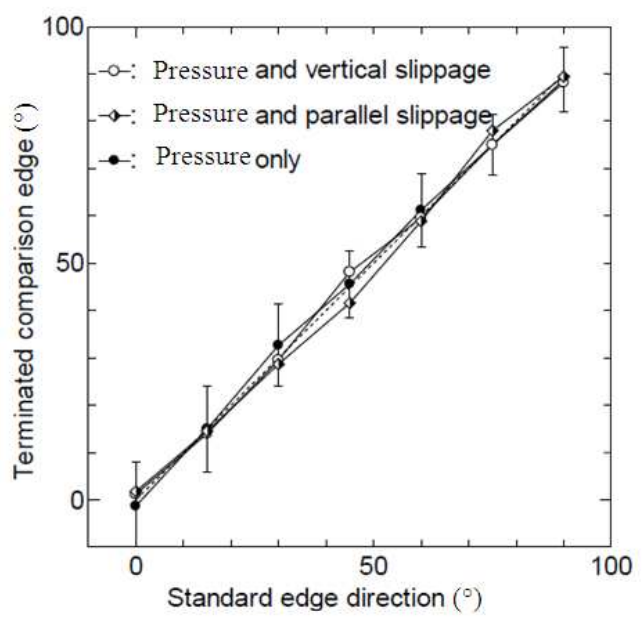

Fig. 12: Experimental result obtained from tactile mouse

Filled circles show the average value of the terminated comparison edge direction. When the standard edge direction equals $0^{\circ}$, the terminated comparison edge direction deviates from the dotted line. However, except for $0^{\circ}$. The terminated comparison edge orientation coincides with the dotted line. Human subjects can adjust the comparison edge direction to the standard edge direction with high precision.

Virtual edge test: Figure 12 shows the experimental result obtained from the tactile mouse. Since we performed this experiment with three conditions, three curves appear in this figure. Although these three curves almost coincide with the dotted line, considerable difference from the dotted line is observed compared to Fig. 11 for the real edge. 


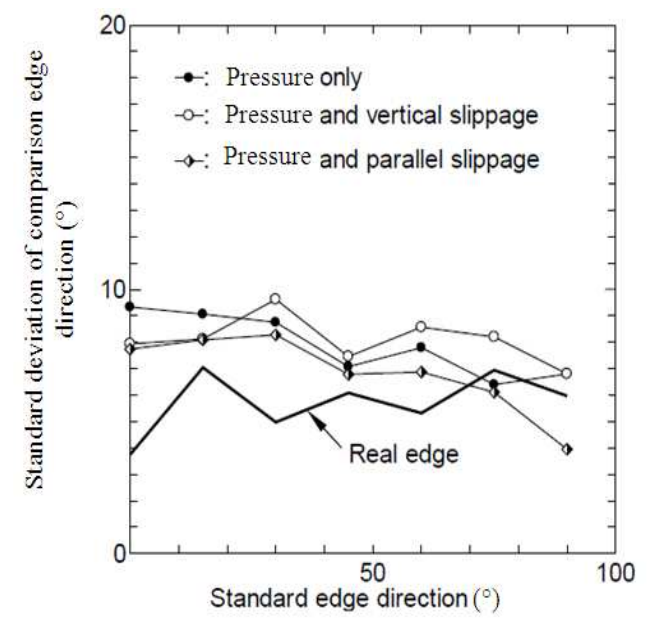

Fig. 13: Comparison between real edge presentation and virtual edge presentation

\section{DISCUSSION}

Since the standard deviation can be treated as the difference threshold in psychophysics, the standard deviation of the comparison edge direction is examined in Fig. 13. The thick solid line shows the result of the real edge, which takes the smallest value under $60^{\circ}$. Since the smaller difference threshold means better precision of sensation, the real edge presentation is beyond the virtual edge presentation with the tactile mouse.

During virtual edge presentations, the case of distributed pressure and parallel slippage force presentation is the smallest. Although the difference between it and the second smallest is not considerably large, it is shown that combined presentation of distributed pressure and slippage force is effective for edge presentation.

On the other hand, the result of distributed pressure and vertical slippage force presentation shows the largest value from $30-90^{\circ}$. Since vertical slippage force makes a human subject's finger move away from the edge, misunderstanding of edge position seems to occur.

\section{CONCLUSION}

To evaluate the advantage of pressure-slippage force presentation, we performed a series of psychophysical experiments using a real edge presentation apparatus and a tactile mouse. Although the combined presentation of distributed pressure and vertical slippage force is rather worse than only distributed pressure presentation, the combined presentation of distributed pressure and parallel slippage force is effective for presenting the virtual edge. The combined presentation seems to be more effective for higher-order recognition such as texture presentation and feeling of material demonstration. In future work, we will tackle these problems.

\section{ACKNOWLEDGEMENTS}

The researchers thank Fuji Xerox for providing the tactile mouse.

\section{REFERENCES}

Arata, S. and Y. Ikei, 2006. Wideband Vibratory Stimulation on the Texture Display 2R. Tokyo Metropolitan University. Bolanowaski, S., J. Gescheider, G.A. Verrillo, R.T. and C.M. Checkosky, 1988, Four channels mediate the mechanical aspects of touch. J. Acoustical Society Am., 84: 1680-1694. DOI: 10.1121/1.397184

Gescheider, G.A., 1997. Psychophysics. The Fundamentals, Lawrence Erlbaum Associates. 3rd Edn., Psychology Press, Routledge, ISBN: 080582281X, 9780805822816

Iwata, H., H. Yano, F. Nakaizumi and R. Kawamura, 2001. Project FEELEX. Adding Haptic Surface to Graphics. Proceedings of the 28th Annual Conference on Computer Graphics and Interactive Techniques, California USA, (SIGGRAPH '01), ACM New York, USA, pp: 469-475. DOI: $10.1145 / 383259.383314$

Konyo, M., S. Tadokoro and T. Takamori, 2000. Artificial tactile feel display using soft gel actuators. IEEE Int. Conf. Robot. Automat., 4: 3416-3421. DOI: 10.1109/ROBOT.2000.845250

Nara, T., M. Takasaki, T. Maeda, H. Higuchi and S. Ando et al., 2001. surface acoustic wave . (SAW) tactile display based on properties of mechanoreceptors. Proceeding of the IEEE Virtual Reality Conference, March 17-17, IEEE Xplore Press, Yokohama, Japan, pp: 13-20. DOI: 10.1109/VR.2001.913765

Ohka, M., H. Koga, Y. Mouri, T. Sugiura and T. Miyaoka et al., 2007. Figure and texture presentation capabilities of a tactile mouse equipped with a display pad of stimulus pins. Robotica, 29: 527-533. $\quad$ DOI: $10.1017 / \mathrm{S} 026357471000041 \mathrm{x}$

Salisbury, J.K. and M.A. Srinivasan, 1997. Phantombased haptic interaction with virtual objects. IEEE Comp. Graph. Appl., 17: 6-10. DOI: 10.1109/MCG.1997.10014 
Shimojo, M., M. Shinohara and Y. Fukui, 1999. human shape recognition performance for 3-d tactile display. IEEE Trans. Sys. Man and Cyberne. Sys. Hum., 4: 3192-3197. DOI: 10.1109/ICSMC.1997.633090

Shinohara, M., Y. Shimuzu and A. Mochizuki, 1998. Three-dimensional tactile display for the blind. IEEE Trans. Rehabili. Eng., 6: 249-256. DOI: $10.1109 / 86.712218$
Tachi, S., 1992. Tele-Existence. J. Robot. Mech., 4: 7-12.

Zhou, Y., C. Yin, M. Ohka and T. Miyaoka, 2010. Tactile display mounted on a mouse. Int. J. Comp. Network Security, 2: 38-42. 